\title{
Application of accelerated time models to compare performance of two comorbidity- adjusting methods with APACHE II in predicting short-term mortality among the critically ill
}

George Mnatzaganian ${ }^{1 *}$, Melanie Bish ${ }^{1}$, Jason Fletcher ${ }^{2}$, Cameron Knott $^{2-5}$, John Stephenson ${ }^{6}$

1. La Trobe Rural Health School, College of Science, Health and Engineering, La Trobe University, Victoria, Australia

2. Intensive Care Unit, Bendigo Health, Barnard Street, Bendigo, Victoria, Australia

3. Monash Rural Health Bendigo, Monash University, Bendigo, Victoria, Australia

4. Department of Intensive Care, Austin Health, Heidelberg, Victoria, Australia

5. Honorary Clinical Fellow, Austin Clinical School, University of Melbourne, Heidelberg, Victoria, Australia

6. School of Human and Health Sciences, University of Huddersfield, Queensgate, Huddersfield, United Kingdom

Running title: Comorbidity indices and mortality among ICU patients

Corresponding author: Dr George Mnatzaganian, College of Science, Health and Engineering, La Trobe Rural Health School, PO Box 199 Bendigo, 3552, Victoria, Australia

T: +61 354447071 | F: +61 354447477 | Email: G.Mnatzaganian@latrobe.edu.au

Co-authors' emails: Dr Melanie Bish: M.Bish@latrobe.edu.au; Dr Cameron Knott: cknott@bendigohealth.org.au; Dr Jason Fletcher: JFletcher@bendigohealth.org.au; Dr John Stephenson: J.Stephenson@hud.ac.uk 


\section{Summary}

\section{Objective}

This study aimed to determine how the abilities of the Charlson Index and Elixhauser comorbidities compared with the chronic health components of the Acute Physiology and Chronic Health Evaluation (APACHE II) to predict in-hospital 30 day mortality among adult critically ill patients treated inside and outside of Intensive Care Unit (ICU).

\section{Methods}

A total of 701 critically ill patients, identified in a prevalence study design on four randomly selected days in five acute care hospitals, were followed up from the date of becoming critically ill for 30 days or until death, whichever occurred first. Multiple data sources including administrative, clinical, pathology, microbiology and laboratory patient records captured the presence of acute and chronic illnesses. The exponential, Gompertz, Weibull, and log-logistic distributions were assessed as candidate parametric distributions available for the modelling of survival data. Of these, the log-logistic distribution provided the best fit and was used to construct a series of parametric survival models.

\section{Results}

Of the 701 patients identified in the initial prevalence study, 637 (90.9\%) had complete data for all fields used to calculate APACHE II score. Controlling for age, sex and Acute Physiology Score (APS), the chronic health components of the APACHE II score, as a group, were better predictors of survival than Elixhauser comorbidities and Charlson Index. Of the APACHE II chronic health components, only the relatively uncommon conditions of liver failure (3.4\%) and immunodeficiency (9.6\%) were statistically associated with inferior patient survival with acceleration factors of $0.35(95 \% \mathrm{Cl} 0.17,0.72)$ for liver failure, and $0.42(95 \% \mathrm{Cl} 0.26,0.72)$ for immunodeficiency. Sensitivity analyses on an imputed dataset that also included the 64 individuals with imputed APACHE II score showed identical results.

\section{Conclusion}

Our study suggests that, in acute critical illness, most co-existing comorbidities are not major determinants of short-term survival, indicating that observed variations in ICU patient 30-day mortality may not be confounded by lack of adjustment to pre-existing comorbidities. 


\section{Keywords}

Accelerated time modelling, APACHE II, Charlson Index, critically ill, Elixhauser comorbidities, in-hospital mortality.

\section{Introduction}

The global burden of critical illness is hard to quantify because of the many epidemiological challenges in measuring the incidence and prevalence of critical disease. Nonetheless, population-based studies suggest a gradual increase in the demand for critical care services as the population ages [1]. The brief and often sudden prodrome of critical illness and its relatively high short-term mortality add to this challenge. One of the main determinants of this short-term survival is the severity of the acute illness [2]. Over the past few decades, various scoring systems have been developed to measure the severity of illness among the critically ill [3], amongst which, the Acute Physiology and Chronic Health Evaluation (APACHE) II remains the most widely used [4-8]. However, patients requiring critical care frequently present with multiple physiological abnormalities and are often relatively old [9] with multiple chronic comorbid conditions that are not captured by APACHE II [10]. It is unreasonable to expect APACHE II to fully account for casemix derived from multiple comorbid conditions [11]. The adjustment for the burden of pre-existing comorbid conditions among the critically ill remains uncertain [12].

Studies that compared the predictive ability of a comorbidity index such as the Charlson Index [13-17] or Elixhauser comorbidities $[12,16]$ with that of the comorbidity components of the APACHE II score have produced conflicting results. Some studies found that comorbidities significantly improved prognostic information independent of that attained from the chronic health points that constitute APACHE II score $[12,14]$. Others did not find any significant improvement in mortality model discrimination when the Charlson Index or number of Elixhauser comorbidities was added to the APACHE II model that did not include its chronic components $[13,15,16]$.

Inconsistencies in the findings of these and other studies may reflect sampling biases, confounded by indication [18] or relatively small samples [14], incomplete adjustment for comorbidities in studies that used administrative databases [12, 13, 15-17], or single-site studies that involved patients that may not have been entirely representative of the critical 
ill population $[13,14]$. Furthermore, critically ill patients who were not treated in intensive care units (ICU) were not included in any of the other studies. Such critically ill patients treated outside of ICU may have been refused admission to ICU based on age, underlying comorbidities, perceived futility due to a high probability of death in spite of admission to ICU [19], or due to shortage in ICU beds [20-22]. Female sex as an independent factor associated with less admission to intensive or coronary care units has also been reported $[23,24]$.

The main objective of this study, which conducted secondary analyses on prevalence data collected in five acute care tertiary hospitals in Israel [20,25], was to compare the in-hospital 30-day mortality predictive abilities of the Charlson Index and Elixhauser comorbidities to those of the chronic health points of the APACHE II score among adult hospitalised critically ill patients. Previous studies targeted the critically ill treated in ICUs. This study targeted all critically ill patients treated in all adult hospital departments regardless of whether they were admitted to an ICU. Patient data from multiple clinical, administrative, pathology and laboratory data sources were utilised to capture acute and chronic illness diagnoses.

\section{Methods}

\section{Study design and data collection}

Study details have been previously described $[20,25]$. In summary, five acute care hospitals in Israel volunteered to participate in the original multi-site prevalence study; three of which were the largest teaching hospitals in the country. On four randomly selected days, all five hospitals were screened to identify critically ill patients in all adult wards. In each of the hospitals, on the same days, a team of intensivists, epidemiologists and nurses visited all adult hospital beds to identify patients meeting ICU admission criteria (study criteria). These criteria were developed by the directors of all ICUs in the country using consensus decision-making that was mainly based on, and guided by, the Task Force of the Society of Critical Care Medicine [26]. Hospital wards were categorised into three types: (i) intensive care units (ICU) which included general and respiratory ICU; (ii) specialised care units that included coronary care units, surgical recovery rooms, neurological and medical intensive special care beds; and (iii) general / surgical or medical wards that included all adult hospital departments that did not have intensive specialised care. All critically ill patients hospitalised within 24 hours preceding the screening, including those who died or were transferred to another facility, 
were eligible to be included in the study. Terminally ill patients with a Do Not Resuscitate (DNR) order were not eligible to participate.

Following the screening process, all information relevant to the study was collected from the patients' medical records, biochemistry and pathology results, microbiological laboratory reports, discharge summaries and hospital administrative databases. Information collected included: demographics, dates of admission and discharge, date of clinical deterioration, acute diagnoses leading to clinical deterioration, presence of infections, all pre-existing comorbidities, treating hospital setting, items needed to estimate the Acute Physiology Score (APS-12) [2] and APACHE II [2,20], and the simplified Therapeutic Intervention Scoring System (TISS-28) [27] that measured intensity of nurse treatment. All-cause 30-day mortality from the time the patient became critically ill was also recorded $[7,20,25]$. The chronic health points of the APACHE II collected in the original study collated cardiovascular and respiratory organ insufficiency as one group; thus, the chronic components included in this study were having liver, renal or cardiovascular/respiratory insufficiency and being immunocompromised.

\section{Construction of Charlson Index and Elixhauser co-morbidities}

Using the International Classification of Diseases, Ninth Edition, Clinical Modification (ICD-9CM) coding algorithms, we calculated the Charlson co-morbidity index and defined Elixhauser comorbidities $[28,29]$ based on all comorbid conditions extracted from all data sources. All ICD-9-CM codes used to define Charlson and Elixhauser comorbidities are presented in Appendices 1 and 2. Charlson Index is based on 17 comorbid conditions, adapted from initial 19 conditions [30]. Using the original Charlson weights used to construct the final score [30], comorbidities were assigned with weights of $1,2,3$, or 6 depending on the condition, which were then summed to form the index. The comorbidities associated with each of these weights are presented in Appendix 1.

Elixhauser comorbidities include a comprehensive set of 31 comorbid conditions [29], denoted $E_{1}$ to $E_{31}$ (Appendix 2). To avoid consideration of variables with very low numbers of events, and to ensure an adequate number of events-per-variable, certain related Elixhauser comorbidities were combined into single measures. A variable derived from Elixhauser comorbidities $i$ and $j$ is denoted as $E_{i, j}$. For power concerns, conditions with very few cases (e.g. past history of peptic disease) were not included as separate comorbid categories. 


\section{Statistical analysis}

All patients were followed from the date of becoming critically ill for 30 days, or until death if this occurred before 30 days. Patients who remained alive at the end of the review period were so recorded. Deaths within 30 days were recorded including the date of death. Both groups of patients in the sample were summarised descriptively.

The exponential, Gompertz, Weibull, and log-logistic distributions were assessed as candidate parametric distributions available for the modelling of survival data, using null models [31]. Of these, the log-logistic distribution was deemed the best fitting survival distribution as it had the lowest value of the Akaike Information Criteria (AIC) statistic (1090.32, compared to 1091.52 for Weibull and Gompertz distributions, and 1090.65 for the exponential distribution). The log-logistic distribution is parameterised in an accelerated failure time metric [32], a general model for survival data in which covariates act multiplicatively on the time scale, leading to an interpretation in terms of the speed of progression of terminal illness amongst critically ill ICU patients, and allowing for a unimodal hazard function [33]. A series of parametric survival models were derived using the selected distribution to determine:

- the capability of the APS score together with the chronic health points that constitute APACHE II score to predict all-cause 30-day patient survival, controlling for sex and age (Model 1);

- whether inclusion of the Charlson index in conjunction with the APS score significantly improved predictive capability from that obtained from the APS score and the chronic health points alone, controlling for sex and age (Model 2); and

- whether inclusion of Elixhauser comorbidities in conjunction with the APS score significantly improved predictive capability from that obtained from the APS score and the chronic health points alone, controlling for sex and age.

Two models were derived: a full model including all possible comorbidities and a parsimonious model including only those comorbidities showing some substantive importance $(p<0.2)$ in the full model (Models $3(a) ; 3(b)$ ). For each model, $p$-values and acceleration factors (calculated as the exponent of the parameter estimate) with associated 95\% confidence intervals were derived for all included covariates together with the likelihood ratio statistic (LRS). 
Survival curves were generated for patients with key values of components of assessed indices appearing to substantively predict survival. Hazard functions for relevant indices were also generated as a clinically meaningful measure to increase the understanding of the nature of the course of disease for patients of varying conditions.

\section{Multiple imputation}

Of the 701 patients, 64 (9.1\%) had a missing APACHE II score. Little's test was used to assess whether this missing was completely at random (MCAR). The 64 missing APACHE II scores were imputed using multiple imputation [34]. The multiple imputation was conducted using chained equations using the mi Stata command, with 50 imputed datasets and final estimates obtained using Rubin's rules [35].

All analyses were undertaken using Stata statistical software (version 14, Stata-Corp.).

\section{Ethical considerations}

This study was conducted in accordance with the Declaration of Helsinki. Ethical approval to conduct the study was granted by the Israeli Ministry of Health and its Human Research Ethics Committee. Because this Ministry of Health, which normally inspects hospitals, conducted the initial study, there was a waiver of informed consent based on Israeli law [36]. Furthermore, all analyses used non-identifiable data.

\section{Results}

Descriptive summary of data

APACHE II scores were available on 637 (90.9\%) of the 701 patients included in the original study [25]. The 64 patients with a missing APACHE II significantly differed from the remaining participants on age, presence of infections, cancer, neurological disorders, TISS scores, and the hospital treating ward with a higher probability of being treated in non-ICU general medical wards. No substantial group differences were observed in the Charlson index, trauma status, sex, most of Elixhauser comorbidities, and 30-day mortality (Table 1).

Accounting for 30-day mortality, sex, Charlson index, admission due to trauma, and most Elixhauser comorbidities (excluding cancer, and neurological disorders), Little's test showed that APACHE II score was missing completely at random (Little's MCAR test $\chi^{2}=8.5, p=0.5$ ). However, when all other study variables were included, missing of APACHE II score was not 
at random (Little's MCAR test $\chi^{2}=92, p<0.001$ ). These 64 patients were excluded from the main analyses.

$\ll$ Please insert Table 1 >

Of the included 637 patients, 181 (28.4\%) died within 30 days of becoming critically ill (time of clinical deterioration). Compared to the survivors, those who died were older $(p<0.001)$ with significantly higher Charlson index $(p=0.01)$ and APACHE II score $(p<0.001)$ (Table 2). Amongst the 637 patients, 12616 days of patient survival were recorded (average of 19.8 days per patient), with deaths being recorded at an incidence of 0.0143 per day. Median death rates could not be determined, as deaths did not reach $50 \%$ of the cohort during the analysis period; $25 \%$ of all deaths occurred within 20 days of onset of critical illness.

$<<$ Please insert table 2>>

A comparison of characteristics amongst patients who did and did not die during the followup period suggested that the APACHE II chronic health points, APS and Charlson scores, and some of the Elixhauser comorbidities, may be likely to be associated with survival, vindicating their selection in this analysis. No substantive association existed between survival and the TISS score, which was not considered further in this analysis.

Analysis of model fit

P-values, acceleration factors and associated 95\% confidence intervals (Cls) for all factors/covariates in each model are summarised in Table 3, alongside the LRS for each model. A model that accounted for age, sex, APS, and the chronic components of the APACHE II score, as a group, was a better predictor of survival than Elixhauser comorbidities and Charlson Index when sex, age and APS were added to each of the latter.

$<<$ Please insert Table 3>>

Survival curves for patients differentiated by the presence or absence of each of the APACHE II chronic health points are shown in Figure 1. All functions are evaluated at mean values of other covariates. Among the chronic points of APACHE II, presence or absence of liver insufficiency or immunodeficiency were most effective to differentiate between patients in terms of survival, however, these conditions accounted for a relatively low proportion of patients in the cohort. Of the chronic health points, cardiovascular or respiratory insufficiency was observed in the highest proportion of patients (26.6\%) but, similar to renal insufficiency, 
appeared to be less effective in differentiating between patients in terms of short-term survival. Incidences of death for patients suffering from each of the chronic health points individually ranged from 0.01205 per day (renal insufficiency) to 0.03658 (liver insufficiency). Frequencies of patients suffering from two or more of these conditions were in general too low to justify generation of incidence rates.

$<<$ Please insert Figure 1 $>>$

Charlson scores were recorded in the range from 0 to 6 , with the majority of patients having an APACHE II score between 10 and 40. Survival graphs plotted for Charlson scores of 0, 2, 4 and 6 revealed better survival in patients with lower Charlson scores over the full analysis period. Incidence rates of deaths ranged from 0.00802 amongst those with Charlson value of 0 to 0.01776 amongst those with Charlson index value of 6 . No significant change in incidence of death in patients with non-zero Charlson scores was observed. Only "renal disease" derived from the Elixhauser comorbidities was statistically significant or substantively related to patient survival in either the full model $3(a)$ or the parsimonious model $3(b)$ (Table 3 ). Controlling for age, sex and APS, Elixhauser comorbidities, as a group, were better predictors of survival in comparison to the Charlson Index.

Examination of hazard functions for the APACHE II chronic health points (Figure 2a) showed that maximum mortality rate for patients with liver insufficiency occurred after about five days. This was later for patients with immunodeficiency. There were minimal changes in the lower mortality rates associated with cardiovascular, respiratory or renal failure beyond approximately 10 days. The unimodal hazard functions associated with liver failure and immunodeficiency arose from the parameterisation of the log-logistic distribution in the accelerated failure time metric. Hazard functions for the key Elixhauser comorbidities (Figure 2b) showed that maximum mortality rate for patients with renal disease occurred at approximately five days.

$<<$ Please insert Figure 2>>

\section{Sub-analysis on imputed data}

Models 1 and 2 were re-run on an imputed data set as a sensitivity analysis; with all parameter estimates revealed to be identical or nearly identical, with negligible difference in the fit of 
models derived from the original and imputed data sets. There were no changes in the inferences of significance of any parameter between the models run on the two datasets.

\section{Discussion}

This multisite study compared the abilities of Charlson Index and Elixhauser comorbidities with those of the chronic components of APACHE II in predicting 30-day mortality among critically ill patients treated inside and outside of intensive care units in five acute care hospitals. The key finding was that both Charlson Index and Elixhauser comorbidities were inferior predictors of 30-day mortality than the chronic components of APACHE II. Additionally, there was no association between the Charlson Index, most of Elixhauser comorbidities, and study short-term outcomes in this critically ill population.

The association of Charlson Index and Elixhauser comorbidities with in-hospital mortality has been repeatedly demonstrated [37-39]. However, similar to other studies [13, 16], we found that both the Charlson and Elixhauser comorbidity-adjustment methods performed worse than APACHE II when predicting short-term mortality in the critically ill. Risk of death among patients with various chronic diseases was low and relatively constant over the study period. General similarity in survival and hazard curves found for sub-groups of patients over two of the APACHE II chronic components and a limited number of Elixhauser conditions suggest that both methods are approximately equally effective in predicting survival; however, controlling for sex, age and APS, the model which also included the chronic health points of the APACHE II index was a better fit to the data than the models which either included the Charlson score, or Elixhauser comorbidities. The effect of a small change in the value of the Charlson Index was not substantive, showing that this index, which includes 17 comorbid conditions, was not associated with short-term survival among the critically ill. Among the chronic components of APACHE II, only liver insufficiency and immunodeficiency (both being relatively rare conditions) had a significant ability to discriminate between hospital 30-day survivors and non-survivors. It is possible that after the critically ill survive past their acute phase illness,

comorbidities become stronger determinants of long-term survival improving mortality prediction over time, as shown in another study that showed that the predictive abilities of Charlson Index and Elixahuser comorbidities increased when assessing long-term outcomes in the critically ill [40]. 
Unlike other studies [15-17], this current study captured acute and chronic conditions using various data sources that included medical and nursing reports, discharge summaries, pathology and microbiological laboratory results, and routinely collected administrative hospital data. The sole use of administrative databases, as conducted by most studies that compared APACHE II to other comorbidity adjustment methods [12, 13, 15-17], may introduce classification biases, and confounding due to the under-coding of comorbidities that characterise most routinely collected administrative databases [41]. Critical illness is challenging to study with only administrative databases as critical illness is not accurately captured by hospital coding [1]. Similarly, such administrative data coding often cannot differentiate acute complications from co-existing comorbidities and therefore the predictive performance of administrative-data-based co-morbidity may largely depend on the accuracy and quality of the routinely collected data [38]. Another strength of this study was the use of the log-logistic distribution, parameterised in the accelerated failure time metric, to describe short-term survival. This analytic method provided greater flexibility than would be obtained by a corresponding distribution parameterised in the proportional hazards metric.

This study has limitations. Availability of data did not allow the investigation of long-term outcomes. Although the inclusion of the critically ill treated inside and outside of ICU was a strength in this study, patients who had had a missing APACHE II score were less likely to be treated in an ICU. They were also more likely to be younger than those without a missing APACHE II score. The younger critically ill patients and those treated outside of ICUs may have been under-represented in this study. Nonetheless, the sub-analysis that used multiple imputation to estimate the missing APACHE showed that the exclusion of the $9 \%$ of patients with missing APACHE II had no effect on model findings.

\section{Conclusions}

In conclusion, this study suggests that, in a population with acute critical illness, adjustment for most pre-existing comorbid conditions does not significantly improve 30-day mortality prediction. This may indicate that adjusting for APACHE II is sufficient and that observed variations in patient short-term mortality may less be confounded by lack of adjustment to pre-existing comorbidities. 


\section{Abbreviations}

AIC, Akaike Information Criteria; APACHE, Acute Physiology and Chronic Health Evaluation; APS, Acute Physiology Score; Cl, confidence interval; DNR, do not resuscitate; ICD-9-CM, international classification of diseases, ninth edition, clinical modification; ICU, intensive care unit; LRS, likelihood ratio statistic; SD, standard deviation; TISS, Therapeutic Intervention Scoring System.

\section{Competing interest}

The authors declare that they have no competing interests.

\section{Funding}

This study had no funding.

\section{Authors' contribution}

GM and JS led the conceptual development of the current study. Subsequent study conception: GM, MB, CK, JF, and JS; Data collection: GM; Analysis of data: GM and JS; Interpretation of findings: GM and JS. Critical revision of draft for intellectual content and approval of final version: $\mathrm{GM}, \mathrm{MB}, \mathrm{CK}, \mathrm{JF}$, and JS. GM serves as the guarantor for the manuscript.

\section{Acknowledgements}

We acknowledge the ICU consortium that included the Department of Health Services Research, Ministry of Health, Israel, and all five hospitals that participated in the original study (Hadassah Hebrew University Medical Centre, Jerusalem; Haemek Hospital, Afula; Rambam Medical Centre, Haifa; Western Galilee Hospital, Naharya; and Soroka University Medical Centre, Beer-Sheva). 


\section{References}

1. Adhikari NKJ, Fowler RA, Bhagwanjee S, Rubenfeld GD. Critical care and the global burden of critical illness in adults. Lancet 2010; 376 (9749): 1339-46.

2. Knaus WA, Draper EA, Wagner DP, Zimmerman JE. APACHE II: a severity of disease classification system. Crit Care Med 1985; 13 (10): 818-29.

3. Bouch CD, Thompson JP. Severity scoring systems in the critically ill. Contin Educ Anaesth Crit Care Pain 2008; 8( 5): 181-185. doi: 10.1093/bjaceaccp/mkn033.

4. Papachristou GI, Muddana V, Yadav D, O’Connell M, Sanders MK, Slivka A, et al. Comparison of BISAP, Ranson's, APACHE-II, and CTSI scores in predicting organ failure, complications, and mortality in acute pancreatitis. Am J Gastroenterol 2010; 105 (2): 435-41. doi:10.1038/ajg.2009.622.

5. Rowan KM, Kerr JH, Major ED, McPherson K, Short A, Vessey MP. Intensive Care Society's Acute Physiology and Chronic Health Evaluation (APACHE II) study in Britain and Ireland: A prospective, multicenter, cohort study comparing two methods for predicting outcome for adult intensive care patients. Crit Care Med 1994; 22 (9): 1392401.

6. Del Bufalo C, Morelli A, Bassein A, Fasano L, Quarta CC, Pcilli AM, et al. Severity scores in respiratory intensive care: APACHE II predicted mortality better than SAPS II. Resp Care 1995; 40 (10): 1042-7.

7. Mnatzaganian G, Sprung C, Zitser-Gurevich Y, Galai N, Goldschmidt N, Levi L, et al. Effect of infections on 30-day mortality among critically ill patients hospitalized in and out of the intensive care unit. Crit Care Med 2008; 36 (4): 1097-104.

8. Simchen E, Sprung C, Galai N, Zister-Gurevich Y, Bar-Lavi Y, Levi L, et al. Survival of critically ill patients hospitalized in and out of intensive care. Crit Care Med 2007; 35 (2): 449-57.

9. Garland A, Olafson K, Ramsey CD, Yogendran M, Fransoo R. Epidemiology of critically ill patients in intensive care units: a population-based observational study. Crit Care 2013; 17 (5): R212. doi: 10.1186/cc13026.

10. Wolff JL, Starfield B, Anderson G. Prevalence, expenditures, and complications of multiple chronic conditions in the elderly. Arch Intern Med 2002; 162 (20): 2269-76. 
11. Goldhill DR, Withington PS. The effect of casemix adjustment on mortality as predicted by APACHE II. Intensive Care Med 1996; 22 (5): 415-9.

12. Johnston JA, Wagner DP, Timmons S, Welsh D, Tsevat J, Render ML. Impact of different measures of comorbid disease on predicted mortality of intensive care unit patents. Med Care 2002; 40 (10): 929-40.

13. Norena M, Wong H, Thompson WD, Keenan SP, Dodek PM. Adjustment of intensive care unit outcomes for severity of illness and comorbidity scores. J Crit Care 2006; 21 (2): $142-50$.

14. Poses RM, McClish DK, Smith WR, Bekes C, Scott WE. Prediction of survival of critically ill patients by admission comorbidity. J Clin Epidemiol 1996; 49 (7): 743-7.

15. Quach S, Hennessy DA, Faris P, Fong A, Quan H, Doig C. A comparison between the APACHE II and Charlson Index Score in predicting hospital mortality in critically ill patients. BMC Health Serv Res 2009; 9: 129. doi: 10.1186/1472-6963-9-129.

16. Ho KM, Finn J, Knuiman M, Webb SAR. Combining multiple comorbidities with Acute Physiology Score to predict hospital mortality of critically ill patients: a linked data cohort study. Anaesthesia 2007; 62 (11): 1095-100.

17. Christensen S, Johansen MB, Christiansen RJ, Lemeshow S. Comparison of Charlson comorbidity index with SAPS and APACHE scores for prediction of mortality following intensive care. Clin Epidemiol 2011; 3: 203-11.

18. Mnatzaganian G, Davidson DC, Hiller JE, Ryan P. Propensity score matching and randomization. J Clin Epidemiol. 2015;68(7):760-768.

19. Pintado MC, Villa P, Gonzalez-Garcia N, Lujan J, Molina R, Trascasa M, et al. Characteristics and outcomes of elderly patients refused to ICU. ScientificWorldJournal 2013; 590837. doi: 10.1155/2013/590837.

20. Simchen E, Sprung C, Galai N, Zitser-Gurevich Y, Bar-Lavi Y, Gurman G, et al. Survival of critically ill patients hospitalized in and out of intensive care units under paucity of intensive care unit beds. Crit Care Med 2004; 32 (8): 1654-61.

21. Carroll GC, Herbert DA. Using population death rate to predict rate of admissions to the intensive care unit. Crit Care Med 2004; 32 (1): 70-6.

22. Franklin C, Rackow EC, Mandoni B, Burke G, Weil MH. Triage considerations in medical intensive care. Arch Intern Med 1990; 150 (7): 1455-9. 
23. Mnatzaganian G, Braitberg G, Hiller JE, Kuhn L, Chapman R. Sex differences in inhospital mortality following a first acute myocardial infarction: symptomatology, delayed presentation, and hospital setting. BMC Cardiovasc Dis 2016; 16 (1): 109. DOI: 10.1186/s12872-016-0276-5.

24. Valentine A, Jordan B, Lang T, Hiesmayr M, Metnitz PGH. Gender-related differences in intensive care: a multiple-center cohort study of therapeutic interventions and outcome in critically ill patients. Crit Care Med 2003; 31 (7): 1901-7.

25. Mnatzaganian G, Galai N, Sprung C, Zitser-Gurevich Y, Mandel M, Ben-Hur D, et al. Increased risk of bloodstream and urinary infections in intensive care unit (ICU) patients compared with patients fitting ICU admission criteria treated in regular wards. J Hosp Infect 2005; 59 (4): 331-42.

26. Task Force of the American College of Critical Care Medicine. Guidelines for intensive care unit admission, discharge, and triage. Crit Care Med 1999; 27 (3): 633-638.

27. Miranda DR, Rijk AD, Schaufedic W. Simplified Therapeutic Intervention Scoring System: the TISS-28-Results from a multicentre study. Crit Care Med 1996; 24 (1): $64-73$.

28. Quan H, Sundararajan V, Halfon P, Fong A, Burnand B, Luthi JC, et al. Coding algorithms for defining comorbidities in ICD-9-CM and ICD-10 administrative data. Med Care 2005; 43 (11): 1130-9.

29. Elixhauser A, Steiner C, Harris DR, Coffey RM. Comorbidity measures for use with administrative data. Med Care 1998; 36 (1): 8-27.

30. Charlson ME, Pompei P, Ales KL, MacKenzie CR. A new method of classifying prognostic comorbidity in longitudinal studies: development and validation. J Chronic Dis 1987; 40 (5): 373-83.

31. Hosmer DW, Lemeshow S, May S. Applied survival analysis. Regression modelling of time-to-event data. 2nd ed. A John Wiley \& Sons, Inc., Publication; 2008.

32. Wei LJ. The accelerated failure time model: A useful alternative to the cox regression model in survival analysis. Stat Med 1992; 11 (14-15): 1871-1879.

33. Lambert $P$, Collett $D$, Kimber A, Johnson R. Parametric accelerated failure time models with random effects and an application to kidney transplant survival. Stat Med 2004; 23 (20): 3177-3192. 
34. Sterne J, White I, Carlin J, Spratt M, Royston P, Kenward M, et al. Multiple imputation for missing data in epidemiological and clinical research: potential and pitfalls BMJ $2009 ; 338$ :b2393.

35. Rubin DB. Multiple Imputation for Nonresponse in Surveys. 99th ed. Michigan: John Wiley \& Sons; 1987.

36. The public health (medical experiments involving human subjects) regulations, 1980. Int Dig Health Legis 1987; 38 (2): 270-2.

37. Librero J, Peiro S, Ordinana R. Chronic comorbidity and outcomes of hospital care: length of stay, mortality, and readmission at 30 and 365 days. J Clin Epidemiol 1999; 52 (3): 171-9.

38. Mnatzaganian G, Ryan P, Hiller JE. Does co-morbidity provide significant improvement on age adjustment when predicting medical outcomes? Methods Inf Med 2014; 53 (2): $115-20$.

39. Frey C, Zhou H, Harvey D, White RH. Co-morbidity is a strong predictor of early death and multi-organ system failure among patients with acute pancreatitis. J Gastrointest Surg 2007; 11 (6): 733-42.

40. Ladha KS, Zhao K, Quraishi SA, Kurth T, Eikermann M, Kaafarani HMA, et al. The DeyoCharlson and Elixhauser-van Walraven comorbidity indices as predictors of mortality in critically ill patients. BMJ Open 2015; 5 (9): e008990. doi: 10.1136/bmjopen-2015008990

41. lezzoni LI, Foley SM, Daley J, Hughes J, Fisher ES, Heeren T. Comorbidities, complications, and coding bias. Does the number of diagnosis codes matter in predicting in-hospital mortality? JAMA 1992; 267 (16): 2197-203. 
Table 1: Patient characteristics of those with and without missing APACHE II score

\begin{tabular}{|c|c|c|c|}
\hline & $\begin{array}{l}\text { With APACHE II } \\
\mathrm{N}=637(90.9 \%)\end{array}$ & $\begin{array}{l}\text { Without APACHE II } \\
\mathrm{N}=64(9.1 \%)\end{array}$ & $p$ value \\
\hline Age in years, mean (SD) & $65.7(18.2)$ & $59.2(17.6)$ & 0.006 \\
\hline Male sex, \% & 54.0 & 53.1 & 0.9 \\
\hline TISS score day $\mathbf{1}^{\wedge}$, mean (SD) & $26.2(11.1)$ & $14.4(6.6)$ & $<0.001$ \\
\hline TISS score day 2 , mean (SD) & $25.2(10.8)$ & $13.5(6.4)$ & $<0.001$ \\
\hline TISS score day $\mathbf{3}$, mean (SD) & $24.2(11.3)$ & $12.3(6.9)$ & $<0.001$ \\
\hline TISS score day 4, mean (SD) & $23.2(11.9)$ & $10.8(8.3)$ & $<0.001$ \\
\hline TISS score day 5 , mean (SD) & $22.5(12.6)$ & $10.3(8.5)$ & $<0.001$ \\
\hline TISS score day 6 , mean (SD) & $21.7(12.7)$ & $9.1(7.3)$ & $<0.001$ \\
\hline \multicolumn{4}{|l|}{$\begin{array}{l}\text { Hospital treating ward on day of clinical } \\
\text { deterioration, } \%\end{array}$} \\
\hline Intensive care unit & 28.9 & 3.1 & \\
\hline Specialised care unit & 24.5 & 24.0 & \\
\hline General medical ward & 46.6 & 78.1 & $<0.001$ \\
\hline $\begin{array}{l}\text { Presence of infection on day of clinical } \\
\text { deterioration, } \%\end{array}$ & 37.5 & 17.2 & 0.001 \\
\hline $\begin{array}{l}\text { Acquired new hospital infection following clinical } \\
\text { deterioration, \% }\end{array}$ & 48.7 & 20.3 & $<0.001$ \\
\hline Charlson index score, mean (SD) & $2.01(1.7)$ & $2.23(1.8)$ & 0.3 \\
\hline Admission due to trauma, $\%$ & 13.7 & 10.9 & 0.5 \\
\hline Selected Elixhauser comorbidities, \% & & & \\
\hline Congestive heart failure & 23.1 & 12.5 & 0.052 \\
\hline Arrhythmia & 23.6 & 18.7 & 0.4 \\
\hline Valvular disease & 10.4 & 6.2 & 0.3 \\
\hline Pulmonary circulation disorders & 2.8 & 1.6 & 0.6 \\
\hline Peripheral vascular disease & 7.5 & 4.7 & 0.4 \\
\hline Hypertension with or without complication & 32.8 & 35.9 & 0.6 \\
\hline Paralysis or other neurological disorders & 25.7 & 10.9 & 0.009 \\
\hline Chronic pulmonary disease & 26.2 & 18.7 & 0.2 \\
\hline Diabetes with or without complications & 7.1 & 3.1 & 0.2 \\
\hline Hypothyroidism & 12.2 & 10.9 & 0.8 \\
\hline Renal disease & 3.0 & 3.1 & 0.9 \\
\hline Liver disease & 1.1 & 1.6 & 0.7 \\
\hline Any cancer (solid / blood / metastatic) / AIDS / HIV & 16.0 & 31.2 & 0.002 \\
\hline Rheumatoid arthritis / collagen disease & 4.9 & 3.1 & 0.5 \\
\hline Coagulopathy & 7.1 & 6.2 & 0.8 \\
\hline Anaemia & 11.9 & 12.5 & 0.9 \\
\hline 30-day mortality, \% & 28.4 & 28.1 & 0.9 \\
\hline
\end{tabular}

${ }^{\wedge}$ The simplified Therapeutic Intervention Scoring System (TISS-28) that measured the intensity of nursing treatment, was collected on six consecutive days from the day of clinical deterioration 
Table 2: Patient characteristics by those who did and did not die within 30 days of becoming critically ill

\begin{tabular}{|l|l|l|l|}
\hline & \multicolumn{2}{|c|}{$\begin{array}{c}\text { Survival within 30 days of } \\
\text { becoming critically ill }\end{array}$} & $P$ value \\
\hline Characteristics & $\begin{array}{l}\text { Survived } \\
\text { N=456 (71.6\%) }\end{array}$ & $\begin{array}{l}\text { Did not survive } \\
\mathbf{N = 1 8 1}(\mathbf{2 8 . 4 \% )}\end{array}$ & \\
\hline Age, mean (SD) & $63.0(18.6)$ & $72.6(15.1)$ & $<0.001$ \\
\hline Female sex, \% & 56.1 & 48.6 & 0.1 \\
\hline Charlson Co-morbidity Index, mean (SD) & $1.9(1.7)$ & $2.3(1.7)$ & 0.01 \\
\hline APACHE II, mean (SD) & $20.6(7.5)$ & $26.2(7.3)$ & $<0.001$ \\
\hline APS, mean (SD) & $14.9(7.3)$ & $18.7(7.3)$ & $<0.001$ \\
\hline TISS, mean (SD) & $26.1(11.1)$ & $26.5(11.0)$ & 0.7 \\
\hline
\end{tabular}

Table 3: P-values, acceleration factors and associated $95 \%$ confidence intervals: all models

\begin{tabular}{|c|c|c|c|c|}
\hline Model & Covariate & $p$ & $\begin{array}{l}\text { Acceleration } \\
\text { factor }\end{array}$ & $\begin{array}{l}95 \% \mathrm{Cl} \text { for } \\
\text { acceleration } \\
\text { factor }\end{array}$ \\
\hline \multirow[t]{8}{*}{1} & Female sex & 0.732 & 1.053 & $(0.783,1.419)$ \\
\hline & Age (continuous) & $<0.001$ & 0.968 & $(0.957,0.979)$ \\
\hline & APS (continuous) & $<0.001$ & 0.948 & $(0.928,0.969)$ \\
\hline & Liver insufficiency & 0.004 & 0.349 & $(0.170,0.718)$ \\
\hline & Cardiorespiratory insufficiency & 0.181 & 0.788 & $(0.557,1.117)$ \\
\hline & Renal insufficiency & 0.374 & 1.466 & $(0.631,3.410)$ \\
\hline & Immunodeficiency & 0.001 & 0.424 & $(0.258,0.697)$ \\
\hline & $L R S=-501.330$ & & & \\
\hline \multirow[t]{5}{*}{2} & Female sex & 0.572 & 1.090 & $(0.808,1.470)$ \\
\hline & Age (continuous) & $<0.001$ & 0.971 & $(0.961,0.982)$ \\
\hline & APS (continuous) & $<0.001$ & 0.951 & $(0.932,0.971)$ \\
\hline & Charlson Index (continuous) & 0.313 & 0.955 & $(0.873,1.044)$ \\
\hline & $L R S=-512.263$ & & & \\
\hline \multirow[t]{16}{*}{ 3(a) } & Female sex & 0.658 & 1.071 & $(0.790,1.454)$ \\
\hline & Age (continuous) & $<0.001$ & 0.969 & $(0.958,0.980)$ \\
\hline & APS (continuous) & $<0.001$ & 0.953 & $(0.934,0.973)$ \\
\hline & $\mathbf{E}_{1}$ [congestive heart failure] & 0.282 & 0.817 & $(0.566,1.180)$ \\
\hline & $\mathrm{E}_{2}$ [cardiac arrhythmias] & 0.891 & 1.024 & $(0.728,1.442)$ \\
\hline & $\mathbf{E}_{3}$ [valvular disease] & 0.253 & 1.360 & $(0.803,2.304)$ \\
\hline & $\begin{array}{l}\mathrm{E}_{4,10} \text { [pulmonary circulation disorders/chronic pulmonary } \\
\text { disease] }\end{array}$ & 0.173 & 1.271 & $(0.900,1.794)$ \\
\hline & $\mathbf{E}_{5}$ [peripheral vascular disease] & 0.232 & 1.475 & $(0.780,2.789)$ \\
\hline & $\mathbf{E}_{6,7}$ [hypertension complicated or uncomplicated] & 0.197 & 1.249 & $(0.891,1.750)$ \\
\hline & $\mathbf{E}_{8,9}[$ paralysis/other neurological disorders] & 0.896 & 1.023 & $(0.733,1.433)$ \\
\hline & $\mathbf{E}_{11,12}$ [diabetes with or without complications] & 0.368 & 1.289 & $(0.728,2.236)$ \\
\hline & $\mathbf{E}_{13}$ [hypothyroidism] & 0.781 & 0.939 & $(0.604,1.459)$ \\
\hline & $\mathbf{E}_{14}$ [renal disease] & 0.014 & 0.391 & $(0.184,0.830)$ \\
\hline & $\mathbf{E}_{15}$ [liver disease] & 0.673 & 0.737 & $(0.179,3.033)$ \\
\hline & $\mathrm{E}_{17,18,19,20}$ [cancer solid or metastatic/HIV/AIDS] & 0.664 & 0.918 & $(0.625,1.349)$ \\
\hline & $\mathrm{E}_{21,22,23,24,25}$ [metabolic disorder] & 0.359 & 0.851 & $(0.603,1.201)$ \\
\hline
\end{tabular}




\begin{tabular}{|c|c|c|c|c|}
\hline & $\mathbf{E}_{26,27}$ [anaemia] & 0.454 & 1.184 & $(0.761,1.840)$ \\
\hline & $\mathbf{E}_{\mathbf{2 8 , 2 9}}$ [substance abuse] & 0.345 & 0.667 & $(0.287,1.547)$ \\
\hline & $\mathbf{E}_{30,31}[$ depression/psychosis] & 0.745 & 1.391 & $(0.197,10.13)$ \\
\hline & $L R S=-502.942$ & & & \\
\hline \multirow[t]{7}{*}{ 3(b) } & Female sex & 0.628 & 1.077 & $(0.798,1.454)$ \\
\hline & Age (continuous) & $<0.001$ & 0.968 & $(0.957,0.978)$ \\
\hline & APS (continuous) & $<0.001$ & 0.952 & $(0.932,0.971)$ \\
\hline & $\begin{array}{l}\mathrm{E}_{4,10} \text { [pulmonary circulation disorders/chronic pulmonary } \\
\text { disease] }\end{array}$ & 0.159 & 1.274 & $(0.910,1.784)$ \\
\hline & $\mathbf{E}_{6,7}$ [hypertension complicated or uncomplicated] & 0.127 & 1.288 & $(0.931,1.781)$ \\
\hline & $\mathbf{E}_{14}[$ renal disease $]$ & 0.013 & 0.387 & $(0.183,0.816)$ \\
\hline & \multicolumn{4}{|l|}{$L R S=-506.708$} \\
\hline
\end{tabular}

Figure 1: Survival curves by APACHE II chronic health components
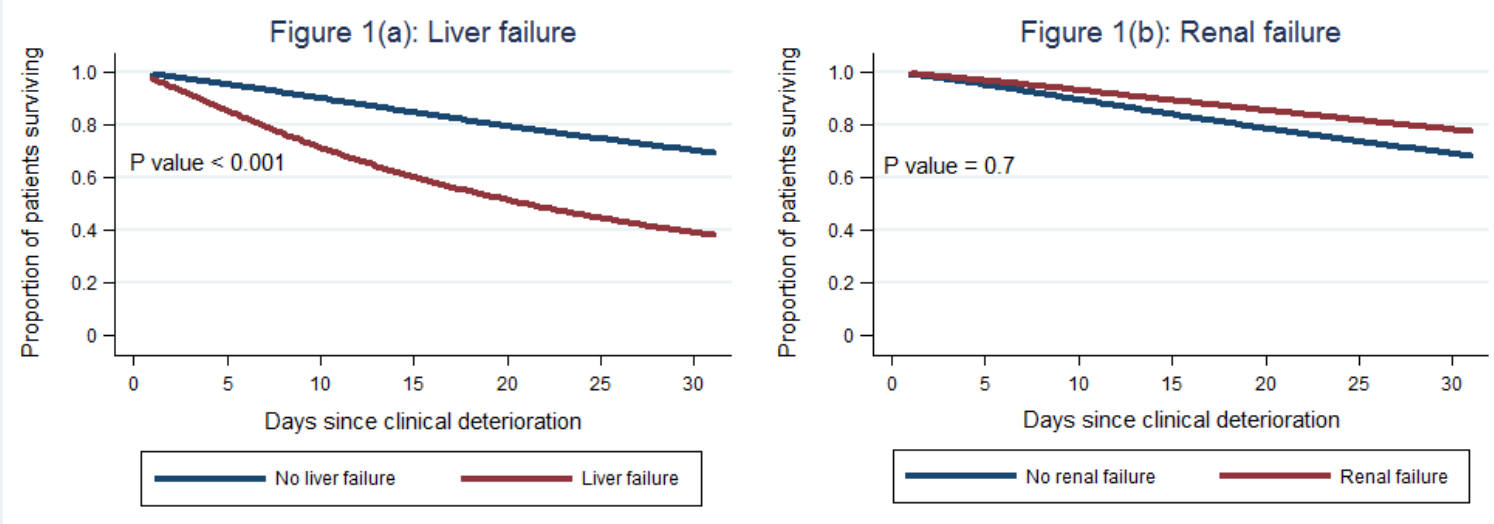

Figure 1(c): Cardio-respiratory failure
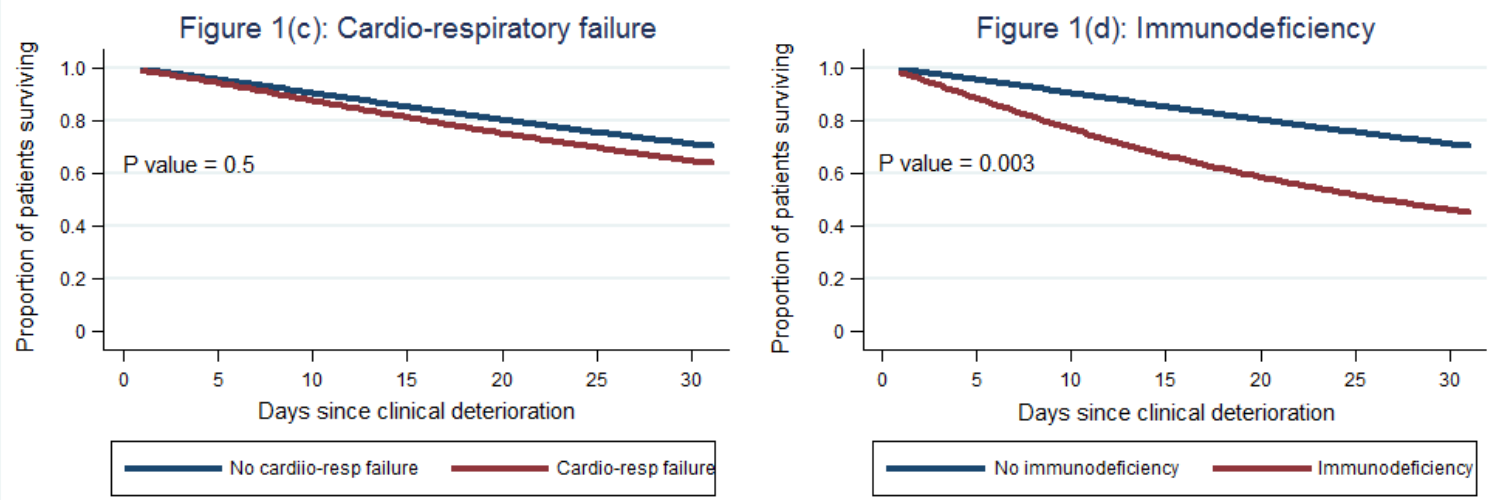
Figure 2: Hazards functions for APACHE II chronic health components and key Elixhauser comorbidities
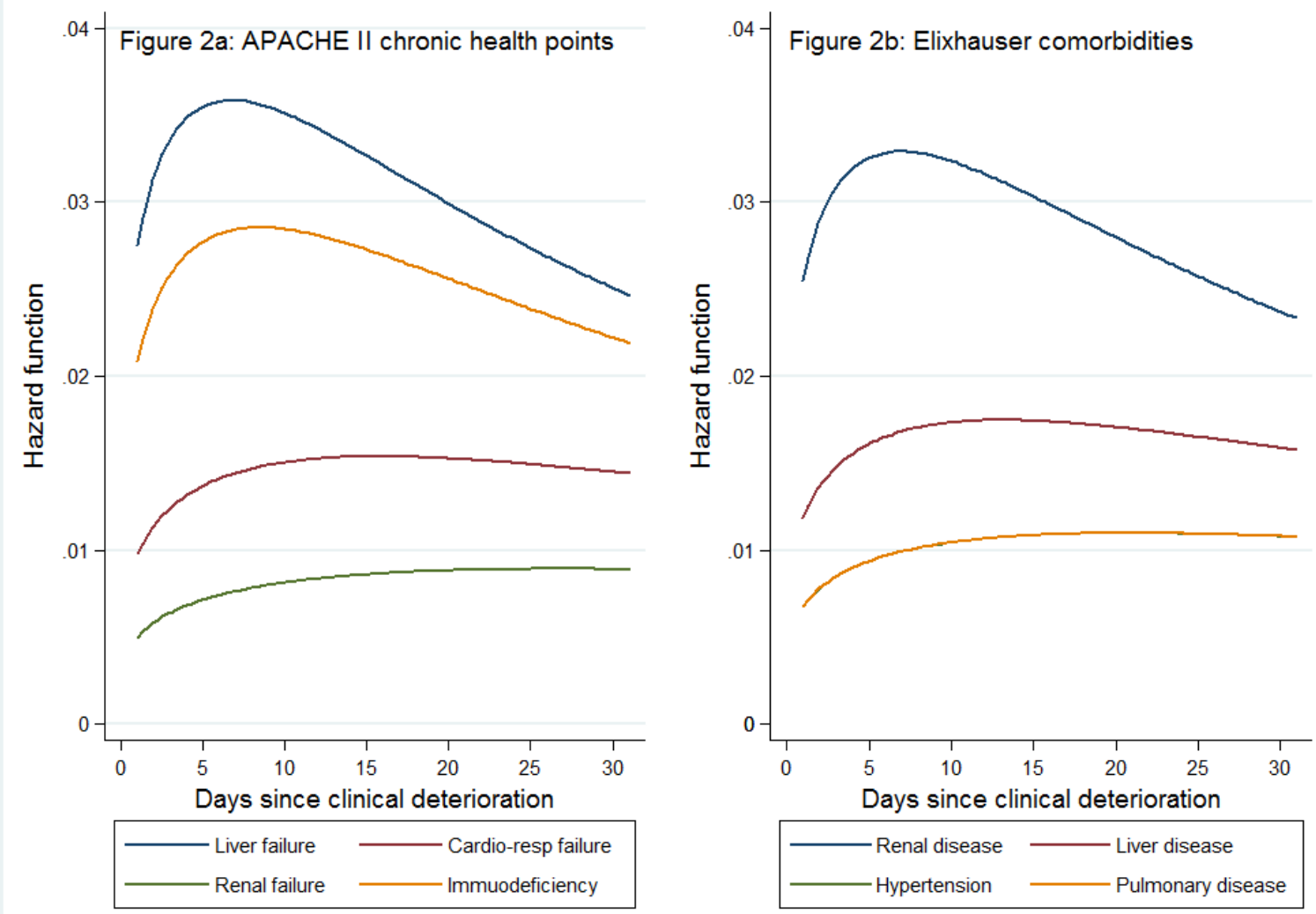